\title{
A REMARK ON DIRECTIONAL CONTRACTIONS
}

\author{
W. A. KIRK ${ }^{1}$ AND WILLIAM O. RAY
}

\begin{abstract}
Let $X$ be a Banach space and $D$ a convex subset of $X$. A mapping $T: D \rightarrow X$ is called a directional contraction if there exists a constant $\alpha \in(0,1)$ such that corresponding to each $x, y \in D$ there exists $\varepsilon=\varepsilon(x, y) \in(0,1]$ for which $\|T(x+\varepsilon(y-x))-T(x)\|<\alpha \varepsilon\|x-y\|$. Tests for lipschitzianness are obtained which yield the fact that if a closed mapping is a directional contraction, then it must be a global contraction, and sufficient conditions are given under which a nonclosed directional contraction $T: D \rightarrow D$ always has a fixed point.
\end{abstract}

Let $X$ be a Banach space and $D$ a convex subset of $X$. It is a well-known and easily proved fact that if $T: D \rightarrow X$ is locally lipschitzian, in the sense that for fixed $k>0$ each point $x$ of $D$ has a neighborhood $U_{x}$ such that $\|T(x)-T(v)\| \leqslant k\|x-v\|$ for each $v \in U_{x}$, then $T$ is globally lipschitzian (with constant $k$ ). In this note we examine substantially weaker local conditions which are sufficient to imply the lipschitzian character of mappings $T: D \rightarrow X$.

Defintion. Let $D$ be a convex subset of $X$. The mapping $T: D \rightarrow X$ is called directionally $k$-lipschitzian for $k>0$ if corresponding to every $x, y \in D$ there exists $\varepsilon=\varepsilon(x, y) \in(0,1]$ such that

$$
\|T(x+\varepsilon(y-x))-T(x)\| \leqslant k \varepsilon\|x-y\| .
$$

If $k \in(0,1)$ then $T$ is called a directional contraction.

M. Altman provided the motivation for this paper when he introduced the notion of directional contractions in [1] and observed that as a special case of his more technical results, if $F: X \rightarrow X$ is a directional contraction for which $P=I-F$ is a closed mapping, then $P(X)=X$. (See [1, \$4].) It is a consequence of our results below that under precisely these assumptions the mapping $F$ is actually a global contraction (from which surjectivity of $P$ is well known). Because directional contractions are global contractions under such mild continuity assumptions, the study of directional contractions as such would appear to be of little importance, but nonetheless we note in our closing remarks that the class of directional contractions which are not contraction mappings is nontrivial and indeed certain mappings of this class always have fixed points.

Received by the editors August 3, 1976 and, in revised form, January 3, 1977.

AMS (MOS) subject classifications (1970). Primary 47H10.

Key words and phrases. Contraction mapping, directional contraction, fixed point theorem.

${ }^{1}$ Research supported in part by a National Science Foundation grant.

- American Mathematical Society 1977 
We begin by giving a relatively weak affirmative test for lipschitzianness of mappings $T: D \rightarrow X$. Recall that such a mapping is closed if for $\left\{x_{n}\right\} \subset D$ the conditions $x_{n} \rightarrow x$ and $T\left(x_{n}\right) \rightarrow y$ imply $x \in D$ and $T(x)=y$.

THEOREM 1. Let $X$ be a Banach space, $D$ a convex subset of $X$, and $T$ : $D \rightarrow X$ a closed mapping. Suppose there exists $k>0$ such that for each distinct $x, y \in D$,

$$
\inf _{\xi \in(0,1]} \frac{\|T(x+\xi(y-x))-T(x)\|}{\xi\|x-y\|} \leqslant k .
$$

Then $T$ is $k$-lipschitzian on all of $D$.

Remark. Clearly if $T$ is directionally $k$-lipschitzian on $D$, then $T$ satisfies (1).

Proof of Theorem 1. Let $x, y \in D$ with $x \neq y$, fix $p>k$, and let $\Omega_{1}$ denote the set of countable ordinals. Suppose for $\gamma \in \Omega_{1}$ and each $\alpha<\gamma$, points $\left\{t_{\alpha}\right\} \subset[0,1]$ have been defined with $t_{0}=0$ so that, if $x_{\alpha}=\left(1-t_{\alpha}\right) x$ $+t_{\alpha} y$, then:

(a) either $t_{\alpha}=1$ for some $\alpha<\gamma$, or $\left\{t_{\alpha}\right\}_{\alpha<\gamma}$ is strictly increasing;

(b) $T$ restricted to the set $\left\{x_{\alpha}: \alpha<\gamma\right\}$ is $p$-lipschitzian.

We define $t_{\gamma}$ as follows: Suppose $\gamma=\beta+1$. If $t_{\beta}=1$ then define $t_{\gamma}=1$; otherwise by (1) (applied to the pair $\left.x_{\beta}, y\right)$ there exists $\bar{x} \in \operatorname{seg}\left(x_{\beta}, y\right]$ such that $\left\|T(\bar{x})-T\left(x_{\beta}\right)\right\| \leqslant p\left\|\bar{x}-x_{\beta}\right\|$, and since $\bar{x}=(1-t) x_{\beta}+t y$ for some $t \in(0,1]$, it follows that $\bar{x}=(1-\bar{t}) x+\overline{t y}$ for $\bar{t} \in\left(t_{\beta}, 1\right]$. In this case define $t_{\gamma}=t$ and observe that for $\alpha \leqslant \beta$, $\left\|T\left(x_{\alpha}\right)-T\left(x_{\beta}\right)\right\| \leqslant p\left\|x_{\alpha}-x_{\beta}\right\|$ and $\left\|T\left(x_{\beta}\right)-T\left(x_{\gamma}\right)\right\| \leqslant p\left\|x_{\beta}-x_{\gamma}\right\|$, from which $\left\|T\left(x_{\alpha}\right)-T\left(x_{\gamma}\right)\right\| \leqslant p \| x_{\alpha}-$ $x_{\gamma} \|$, i.e., $T$ is $p$-lipschitzian on $\left\{x_{\alpha}\right\}_{\alpha \leqslant \gamma}$. Now suppose $\gamma$ is a limit ordinal. In this case define $t_{\gamma}=\sup _{\alpha<\gamma} t_{\alpha}$. To see that $T$ restricted to $\left\{x_{\alpha}\right\}_{\alpha<\gamma}$ is $p$-lipschitzian, observe that for $\alpha<\beta<\gamma$, (b) implies

$$
\left\|T\left(x_{\alpha}\right)-T\left(x_{\beta}\right)\right\| \leqslant p\left\|x_{\alpha}-x_{\beta}\right\| ;
$$

thus, since $\lim _{\beta<\gamma} x_{\beta}=x_{\gamma}$ it follows that $\left\{T\left(x_{\beta}\right)\right\}_{\beta<\gamma}$ is a Cauchy net. By completeness there exists $z \in X$ such that $\lim T\left(x_{\beta}\right)=z$ and since $T$ is a closed mapping, $z=T\left(x_{\gamma}\right)$. Taking limits with respect to $\beta$ in (2), we obtain $\left\|T\left(x_{\alpha}\right)-T\left(x_{\gamma}\right)\right\| \leqslant p\left\|x_{\alpha}-x_{\gamma}\right\|$ for all $\alpha<\gamma$ and thus $T$ restricted to $\left\{x_{\alpha}\right\}_{\alpha<\gamma}$ is also in this case $p$-lipschitzian. Therefore the set $\left\{t_{\gamma}\right\} \subset[0,1]$ is defined with conditions (a) and (b) satisfied for all $\gamma \in \Omega_{1}$. Since $[0,1]$ does not contain a discrete uncountable set, (a) implies that $t_{\gamma}=1$ for some $\gamma \in \Omega_{1}$ from which $x_{\gamma}=y$ and

$$
\|T(x)-T(y)\|=\left\|T\left(x_{0}\right)-T\left(x_{\gamma}\right)\right\| \leqslant p\|x-y\| .
$$

Since $p>k$ is arbitrary, $\|T(x)-T(y)\| \leqslant k\|x-y\|$, completing the proof.

If the constant $k$ in Theorem 1 is less than 1 then an even stronger result holds.

Theorem 2. Let $X$ be a Banach space, $D$ a convex subset of $X$, and $T$ : 
$D \rightarrow X$ a mapping which satisfies (1) of Theorem 1 for $k \in(0,1)$. Suppose the mapping $P=I-T$ satisfies the condition: For each sequence $\left\{x_{n}\right\} \subset D$ such that $x_{n} \rightarrow x_{0} \in D$ the set $\left\{P\left(x_{n}\right)\right\}$ is closed in $X$. Then $T$ is a contraction mapping with global Lipschitz constant $k$ on $D$.

Corollary. With $X, D$, and $T: D \rightarrow X$ as in Theorem 2, if $P=I-T$ maps closed sets into closed sets, then $T$ is a contraction mapping on $D$ with Lipschitz constant $k$.

Proof of Theorem 2. Letting $p \in(k, 1)$, the proof of this theorem follows the proof of Theorem 1 except in defining $t_{\gamma}$ for $\gamma$ a limit ordinal. Suppose $\left\{t_{\alpha}\right\}$ and $\left\{x_{\alpha}\right\}$ satisfying (a) and (b) have been defined for all $\alpha<\gamma$ and let $\alpha_{n} \uparrow \gamma$ as $n \rightarrow \infty$. If $t_{\alpha}=1$ for some $\alpha<\gamma$ then by (b) $\|T(x)-T(y)\| \leqslant$ $p\|x-y\|$. Otherwise by (a), $\left\{t_{\alpha_{n}}\right\}_{n=1}^{\infty}$ is strictly increasing. Define $t_{\gamma}=\lim _{n} t_{\alpha_{n}}$ and suppose $m \neq n$. Then

$$
x_{\alpha_{n}}-T\left(x_{\alpha_{n}}\right) \neq x_{\alpha_{m}}-T\left(x_{\alpha_{m}}\right)
$$

since otherwise

$$
\begin{aligned}
\left\|x_{\alpha_{n}}-x_{\alpha_{m}}\right\| & =\left\|x_{\alpha_{n}}-T\left(x_{\alpha_{n}}\right)+T\left(x_{\alpha_{n}}\right)-T\left(x_{\alpha_{m}}\right)+T\left(x_{\alpha_{m}}\right)-x_{\alpha_{m}}\right\| \\
& =\left\|T\left(x_{\alpha_{n}}\right)-T\left(x_{\alpha_{m}}\right)\right\| \leqslant p\left\|x_{\alpha_{n}}-x_{\alpha_{m}}\right\|,
\end{aligned}
$$

which (since $p<1)$ contradicts $x_{\alpha_{n}} \neq x_{\alpha_{m}}$. Since $\left\|T\left(x_{\alpha_{n}}\right)-T\left(x_{\alpha_{m}}\right)\right\|<p \| x_{\alpha_{n}}$ $-x_{\alpha_{m}} \|,\left\{T\left(x_{\alpha_{n}}\right)\right\}_{n=1}^{\infty}$ is a Cauchy sequence and thus $T\left(x_{\alpha_{n}}\right) \rightarrow y_{0} \in X$. In view of (3), for $N$ sufficiently large,

$$
x_{\gamma}-y_{0} \neq x_{\alpha_{n}}-T\left(x_{\alpha_{n}}\right), \quad n>N .
$$

But since $x_{\alpha_{n}}-T\left(x_{\alpha_{n}}\right) \rightarrow x_{\gamma}-y_{0}$ it follows from our hypothesis on $P$ that

$$
x_{\gamma}-y_{0} \in \bigcup_{n=N}^{\infty}\left\{x_{\alpha_{n}}-T\left(x_{\alpha_{n}}\right)\right\} \cup\left\{x_{\gamma}-T\left(x_{\gamma}\right)\right\}
$$

from which $x_{\gamma}-y_{0}=x_{\gamma}-T\left(x_{\gamma}\right)$, i.e., $y_{0}=T\left(x_{\gamma}\right)$ and $T\left(x_{\alpha_{n}}\right) \rightarrow T\left(x_{\gamma}\right)$. Hence for $\alpha<\gamma$,

$$
\begin{aligned}
\left\|T\left(x_{\alpha}\right)-T\left(x_{\gamma}\right)\right\| & =\lim _{n}\left\|T\left(x_{\alpha}\right)-T\left(x_{\alpha_{n}}\right)\right\| \\
& \leqslant \lim _{n} p\left\|x_{\alpha}-x_{\alpha_{n}}\right\|=p\left\|x_{\alpha}-x_{\gamma}\right\|,
\end{aligned}
$$

proving $T$ is $p$-lipschitzian on $\left\{x_{\alpha}\right\}_{\alpha<\gamma}$. The proof is now completed exactly as in Theorem 1.

We conclude with some remarks about directional contractions. The mapping $T:[0,1] \rightarrow[0,1]$ defined by

$$
T(x)= \begin{cases}1 & \text { if } x \text { is rational, } \\ 0 & \text { if } x \text { is irrational, }\end{cases}
$$

provides a quick example showing that directional contractions are not always contractions, hence that some additional assumption is needed for the validity of Theorem 2 . As we shall see later, it is also the case that directional contractions exist which are not contractions but which satisfy all the 
assumptions of the following theorem. (Of course Theorem 2 precludes the existence of 'natural' examples of this type.)

TheOREM 3. Let $D$ be a closed and convex subset of the Banach space $X$, suppose $T: D \rightarrow D$ is a directional contraction, and suppose the mapping $\eta$ : $D \rightarrow R^{+}$defined by $\eta(x)=\|x-T(x)\|$ is lower semicontinuous. Then $T$ has $a$ fixed point in $D$.

Proof. We follow the approach of Caristi [3]. Suppose $T$ has no fixed point. Then it is possible to define a fixed-point free mapping $g: D \rightarrow D$ as follows: For each $x \in D$ use the directional contraction assumption to select $g(x) \in \operatorname{seg}(x, T(x)]$ so that for fixed $k \in(0,1)$,

$$
\|T(x)-T(g(x))\| \leqslant k\|x-g(x)\| .
$$

Thus

$$
\begin{aligned}
\|x-g(x)\| & =\|x-T(x)\|-\|g(x)-T(x)\| \\
& \leqslant\|x-T(x)\|-[\|g(x)-T(g(x))\|-\|T(g(x))-T(x)\|] \\
& \leqslant\|x-T(x)\|-\|g(x)-T(g(x))\|+k\|g(x)-x\| .
\end{aligned}
$$

From this,

$$
\begin{aligned}
\|x-g(x)\| & \leqslant(1-k)^{-1}[\|x-T(x)\|-\|g(x)-T(g(x))\|] \\
& =\varphi(x)-\varphi(g(x))
\end{aligned}
$$

with $\varphi: D \rightarrow R^{+}$defined by $\varphi(x)=(1-k)^{-1} \eta(x)$. Since $\varphi$ is lower semicontinuous, Caristi's theorem (see [3], [4], [6]) implies $g$ has a fixed point, contrary to our initial assumption.

Remarks. 1. The assumption needed in Theorem 3 is actually considerably weaker than the assumption that $T$ be a directional contraction. One only need suppose that for each $x \in D$ with $x \neq T(x)$ there exists $z \in \operatorname{seg}(x$, $T(x)$ ] such that $\|T(x)-T(z)\| \leqslant k\|x-z\|$.

2. For an example of a directional contraction which satisfies the assumptions of Theorem 3 but which is not a global contraction let $\mathbf{C}$ be the complex plane and suppose $T_{1}$ and $T_{2}$ are distinct contraction mappings of $\mathrm{C} \rightarrow \mathrm{C}$ such that $\left\|z-T_{1}(z)\right\|=\left\|z-T_{2}(z)\right\|$ for all $z \in \mathbf{C}$. (For example, let $T_{1}(z)=z e^{\pi i / 2} / 2, T_{2}(z)=z e^{-\pi i / 2} / 2$.) Let

$$
S=\{z \in \mathbf{C}|| z \mid \text { is rational }\}
$$

and define $T: \mathrm{C} \rightarrow \mathrm{C}$ by

$$
T(z)= \begin{cases}T_{1}(z) & \text { if } z \in S, \\ T_{2}(z) & \text { if } z \notin S .\end{cases}
$$

Clearly $T$ is not continuous, yet $\eta(z)=\|z-T(z)\|$ is continuous (because $\left.\eta(z) \equiv\left\|z-T_{1}(z)\right\|\right)$. To see that $T$ is a directional contraction, one only need consider the case $z_{1} \in S, z_{2} \notin S$. As $\lambda$ ranges over $(0,1),\left|\lambda z_{1}+(1-\lambda) z_{2}\right|$ must assume all intermediate values between $\left|z_{1}\right|$ and $\left|z_{2}\right|$ and, in particular, 
for some point $w=\lambda z_{1}+(1-\lambda) z_{2},|w|$ is rational. Hence

$$
|T(z)-T(w)|=\left|T_{1}(z)-T_{1}(w)\right|<\frac{1}{2}\left|z_{1}-z_{2}\right| \text {. }
$$

The case $z_{1} \notin S$ and $z_{2} \in S$ is similar.

3. By making obvious modifications in the definition and proof it is possible to formulate Theorem 1 assuming only that $D$ is a convex subset of a normed linear space with $T: D \rightarrow M$ a closed mapping of $D$ into a complete metric space $M$.

4. Caristi's theorem used in the proof of Theorem 3 is essentially equivalent to a theorem of I. Ekeland [5] and an elegant proof is implicit in the ideas of A. Brøndsted [2].

\section{REFERENCES}

1. M. Altman, Contractor directions, directional contractors and directional contractions for solving equations, Pacific J. Math. 62 (1970), 1-18.

2. A. Brondsted, On a lemma of Bishop and Phelps, Pacific J. Math. 55 (1974), 335-341.

3. J. Caristi, Fixed point theorems for mappings satisfying inwardness conditions, Trans. Amer. Math. Soc. 215 (1976), 241-251.

4. J. Caristi and W. A. Kirk, Geometric fixed point theory and inwardness conditions, Proc. Conf. on Geometry of Metric and Linear Spaces, Lecture Notes in Math., vol. 490, Springer-Verlag, Berlin and New York, 1975, pp. 75-83.

5. I. Ekeland, Sur les problemes variationnels, C. R. Acad. Sci. Paris 275 (1972), 1057-1059.

6. W. A. Kirk and J. Caristi, Mapping theorems in metric and Banach spaces, Bull. Acad. Polon. Sci. Sér. Sci. Math. Astronom. Phys. 23 (1975), 891-894.

7. W. A. Kirk, Caristi's fixed point theorem and metric convexity, Colloq. Math. 36 (1976), 81-86.

Department of Mathematics, University of Iowa, Iowa Ctry, Iowa 52242 